\title{
MEMORY TECHNOLOGY
}

\section{Watching anti-ferroelectrics switch for VLSI}

In Proc. 2020 IEEE Symposium on VLSI Technology

(in the press); https://go.nature.com/38FQLhw

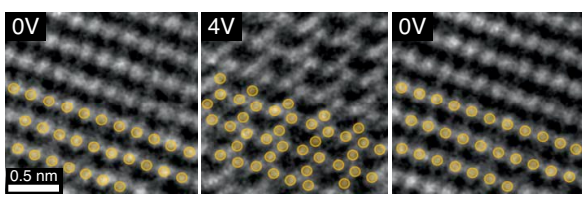

Credit: IEEE

The microstructural properties, and thus the electrical characteristics, of ferroelectric and anti-ferroelectric materials can be controlled by an electric field. As a result, the materials are expected to be of use in several emerging very-large-scale integration (VLSI) technologies, including multilevel non-volatile memory. However, before these materials can be deployed reliably in large-scale systems, a detailed understanding of the relationship between their structural and electrical properties is needed. Sarah Lombardo, Asif Khan and colleagues have now explored one candidate material anti-ferroelectric zirconia $\left(\mathrm{ZrO}_{2}\right)$.

The researchers - who are based at institutes in the US, Germany and Chile used in situ high-resolution transmission electron microscopy to study the nature of electric-field-driven polarization switching in $\mathrm{ZrO}_{2}$ at the atomic scale. The materials were grown using atomic layer deposition to form a metal-insulator-metal structure, with the top metal layer being etched away to allow probing access. The devices operated between $0 \mathrm{~V}$ and $4 \mathrm{~V}$ with cycling endurances of $10^{7}$. Individual grains were observed in situ and throughout four switching cycles. The domain evolution within single grains was tracked showing how three domains merge into to two, and then back to three during a complete biasing cycle. Partial phase transformations were also identified, providing valuable insight that could help in the development of future VLSI applications.

\section{Stuart Thomas}

Published online: 21 July 2020

https://doi.org/10.1038/s41928-020-0453-5 